\title{
The Rotary Cell Culture System increases NTRK3 expression and promotes neuronal differentiation and migratory ability of neural stem cells cultured on collagen sponge
}

\section{Yi Cui}

National Research Institute for Family Planning

Yanyun Yin

Institute of Genetics and Developmental Biology CAS: Institute of Genetics and Developmental Biology Chinese Academy of Sciences

\section{Yannan Zhao}

Institute of Genetics and Developmental Biology CAS: Institute of Genetics and Developmental Biology Chinese Academy of Sciences

Jin Han

Institute of Genetics and Developmental Biology CAS: Institute of Genetics and Developmental Biology Chinese Academy of Sciences

\section{Bai Xu}

Institute of Genetics and Developmental Biology CAS: Institute of Genetics and Developmental Biology Chinese Academy of Sciences

\section{Yunlong Zou}

China-Japan Union Hospital of Jilin University

\section{Bing Chen}

CAS Institute of Genetics and Developmental Biology: Institute of Genetics and Developmental Biology Chinese Academy of Sciences

\section{Zhifeng Xiao}

CAS Institute of Genetics and Developmental Biology: Institute of Genetics and Developmental Biology Chinese Academy of Sciences

\section{Hongwei Song}

EHBIO gene technology

\section{Ya Shi}

CAS Institute of Genetics and Developmental Biology: Institute of Genetics and Developmental Biology Chinese Academy of Sciences

\section{Weiwei Xue}

CAS Institute of Genetics and Developmental Biology: Institute of Genetics and Developmental Biology Chinese Academy of Sciences 


\section{Xu Ma}

National Research Institute for Family Planning

Jianwu Dai ( $\nabla$ xda_dai@genetics.ac.cn )

Institute of Genetics and Developmental Biology Chinese Academy of Sciences

\section{Research}

Keywords: RCCS, NSCs, NTRK3, let-7i-5p, collagen sponge scaffold

Posted Date: February 4th, 2021

DOI: https://doi.org/10.21203/rs.3.rs-115776/v2

License: (c) (1) This work is licensed under a Creative Commons Attribution 4.0 International License. Read Full License 


\section{Abstract}

Background: Recently, neural stem cell (NSC) therapy has shown promise for the treatment of many neurological diseases. Enhancing the quality of implanted cells and improving therapeutic efficacy are currently research hotspots. In this study, we attempted to evaluate whether the combination of 3D cell culturing with the RCCS is the optimal method to improve the neural differentiation ability of NSCs and produce appropriate cells for treating neurological diseases.

Methods: We performed mRNA and miRNA sequencing to characterize NSCs cultured on a collagen sponge in the RCCS. The luciferase assay and Western blot revealed a direct regulatory role between let7i-5p and neurotrophic receptor tyrosine kinase 3 (NTRK3). The neural differentiation markers Tuj1 and Map2 were detected by immunofluorescence staining, and the migratory ability of NSCs was detected both in vitro and in spinal cord injury animals.

Results: NTRK3 expression was elevated in NSCs cultured on collagen sponge in the RCCS. Furthermore, increased NTRK3 expression was regulated by the downregulation of let-7i-5p. Compared with traditionally cultured NSCs, the NSCs cultured on collagen sponge in the RCCS exhibited better neuronal differentiation and migratory ability, especially in the presence of NT-3.

Conclusions: As the biological properties and quality of transplanted cells are critical for therapeutic success, the RCCS combined with collagen sponge shows promise for clinical applications in the future.

\section{Background}

Recently, the transplantation of neural stem cells (NSCs) for the treatment of nervous system diseases has gained attention in neuroscience research[1-3]. NSCs are capable of self-renewal and differentiation and thus hold great promise for nervous system regeneration. As an appropriate cell source for transplantation, NSCs have always been considered suitable for the treatment of many neurodegenerative diseases and traumatic injuries, such as Alzheimer's disease, Parkinson's disease, traumatic brain injury, and spinal cord injury[4-8]. However, the major concern regarding cell transplantation is that many implanted NSCs differentiate into glial cells but not functional neuronal cells. Therefore, discovering methods to overcome these obstacles is crucial[$[9,10]$.

Our previous studies have demonstrated that the conditions in outer space promote neuronal differentiation of NSCs[11]. As microgravity is one of the key factors of outer space[12,13], this present study investigated the influence of simulated microgravity on three-dimensional (3D) cultured NSCs with a dynamic 3D Rotary Cell Culture System (RCCS). The microgravity environment within the RCCS bioreactor is provided by using a random positioning machine that produces low fluid shear force. It is based on continuous rotation of the culture chamber around its horizontal axis, avoiding drops to the bottom of the chamber[14-16]. Here, we selected the collagen sponge scaffold as the cell carrier since it provides a good microenvironment for cell growth and differentiation. The porous network structure of the collagen sponge scaffold helps transport cellular nutritive substances and metabolic waste[17-19]. 
Moreover, regarding animal experiments, a single injection of NSCs may be washed away from the transplantation site by blood, tissue fluid, or cerebrospinal fluid. Conversely, when cells are anchored to the scaffold, NSCs can be easily placed at the injury site.

In this study, we detected that NTRK3 (neurotrophic receptor tyrosine kinase 3) expression was elevated in 3D cultured NSCs in a microgravity environment. The therapeutic potential of neurotrophin-3 (NT-3) has been extensively studied in central nervous system (CNS) injury models[20, 21]. The protective effect of NT-3 was mainly mediated via activation of NTRK3, which may bind specifically and with high affinity to NT-3. Exogenous application of NT-3 has the potential to create a growth-permissive environment for the treatment of neurological disorders[22-24]. Therefore, the expression level of NTRK3 is related to the role and effect of NT-3. The elevated expression of NTRK3 in 3D cultured NSCs in a RCCS bioreactor might improve the positive role of NT-3 on NSCs.

Our study has investigated the molecular mechanisms by which the RCCS affects the biological characteristics of NSCs compared with traditional static culture systems. Furthermore, whether elevated NTRK3 expression in microgravity culture conditions may facilitate neuronal differentiation and the migratory ability of NSCs was assessed. Overall, 3D cultured NSCs in RCCSs may provide an attractive solution for therapeutic cell therapy that could improve the therapeutic effects of transplanted NSCs.

\section{Methods}

NSC isolation and culture

In accordance with a previous report[25], brain hemispheres were dissected and separated by the trypsin enzyme-digesting technique in newborn rats (within $12 \mathrm{~h}$ after birth). All animal procedures were performed in accordance with the Chinese Ministry of Public Health Guide for the care and use of laboratory animals. Single cells were placed in growth medium with B27 supplement (Invitrogen, Carlsbad, CA, www.invitrogen.com), epidermal growth factor $(20 \mathrm{ng} / \mathrm{ml}$, Sigma-Aldrich), and basic fibroblast growth factor $(20 \mathrm{ng} / \mathrm{ml}$, Sigma-Aldrich). After 7 days, the NSCs proliferated and formed neurospheres and were harvested and digested by TrypLE ${ }^{\mathrm{TM}}$ Express Enzyme. For 3D cultures, $1 \times 10^{6} \mathrm{NSCs}$ were seeded onto each collagen sponge scaffold. The next day, the adhesion medium was replaced with traditional differentiation medium with B27 supplement (Invitrogen, Carlsbad, CA, www.invitrogen.com) and without growth factors. The 3D cultured cells were divided into four groups: static (cultured in traditional differentiation medium), RCCS (cultured in traditional differentiation medium), static+NT-3 (cultured in differentiation medium with NT-3 $(50 \mathrm{ng} / \mathrm{ml})$, Peprotech Inc., CA, USA), and RCCS+NT-3 (cultured in differentiation medium with NT-3 $(50 \mathrm{ng} / \mathrm{ml})$, Peprotech Inc., CA, USA). The 3D cultured cells were harvested for subsequent analysis after 12 days in culture.

Animal experiments

Sprague-Dawley rats (200-250 g) were purchased from Charles River Laboratories (Beijing). All animal experimental procedures were performed in accordance with the Chinese Ministry of Public Health guide 
for the care and use of laboratory animals. The SD rats were anesthetized with intraperitoneal injections of ketamine $(80 \mathrm{mg} / \mathrm{kg})$ and xylazine $(10 \mathrm{mg} / \mathrm{kg})$. Following the removal of the vertebral lamina, the T10 spinal cord was exposed and cut off completely using microsurgery scissors. A total length of $1 \mathrm{~mm}$ of spinal cord was removed, and the injury defect was bridged by collagen scaffold with or without cells. The cells that were implanted into the animal models were transfected with the green fluorescent protein (GFP) recombinant adenovirus vector. Bladder emptying and antibiotic treatment were performed routinely after surgery. The rats were sacrificed 2 weeks after surgery for immunofluorescent staining.

Preparation of collagen sponge scaffolds

As previously reported[17], the collagen sponge scaffold was processed from bovine cancellous bone, which had been demonstrated to be an ideal scaffold for culturing cells. The collagen sponge scaffold was $1 \mathrm{~mm}$ thick and $5 \mathrm{~mm}$ in diameter. The surface appearance of the collagen sponge scaffold was observed by a scanning electron microscope (SEM) (S-3000N; Hitachi, Tokyo, Japan).

SEM analysis

To assess cell morphology, the 3D cultured cells were fixed in $2 \%$ glutaraldehyde at $4{ }^{\circ} \mathrm{C}$ overnight and then dehydrated with different concentrations of ethanol. Following conventional approaches and critical point drying, samples were sprayed with gold and then evaluated under SEM (S-3000N; Hitachi).

Fluorescein diacetate NSC staining

Fluorescein diacetate (FDA) can be used to assess the cell viability of numerous cell types, as it generates observable differences in the fluorescence produced by live versus dead cells[26]. The collagen scaffold with implanted cells was incubated with $100 \mu \mathrm{g} / \mathrm{ml}$ FDA for $1 \mathrm{~min}$ in the dark and then rinsed with phosphate-buffered saline. Samples were observed using the Zeiss 200 inverted fluorescent microscope (Carl Zeiss, Jena, Germany).

The miRNA-mRNA regulatory network

RNA sequencing was performed by Novogene company (Beijing, China). Differentially expressed mRNAs were screened by a cut-off fold-change of $\geq 1.5$ and $P$ value of $<0.01$ (Student's $t$-test). The predicted interplay network between these differentially expressed mRNAs and miRNAs involved in regulating them was constructed using Cytoscape (version 3.0.1). The target gene of miRNA was predicted by TargetScan (release 7.0).

Luciferase reporter assays

We performed luciferase assays according to the manufacturer's instructions. To construct the let-7i reporter plasmid, the wild and mutant fragment of the NTRK3 3'-UTR (160 bp) gene containing the putative let-7i binding site was cloned into the pmirGLO Dual-Luciferase miRNA Target Expression Vector (Promega, Madison, WI, USA). The inserted gene segments were synthesized by Generay Biotechnology 
(Shanghai, China). Hela cells were seeded onto 96-well plates and transfected using Lipofectamine $3000^{\text {TM }}$ reagent (Invitrogen, Carlsbad, CA, USA). Firefly luciferase vector $(0.1 \mu \mathrm{g})$ and let-7i-5p mimics (2.5 $\mathrm{nM}$ ) or let-7i-5p inhibitors ( $2.5 \mathrm{nM}$ ) were added to each well. After $48 \mathrm{~h}$, firefly and Renilla luciferase activities were measured by dual-luciferase assays (Promega, Madison, WI, USA). All luciferase data were calculated as a normalized ratio of luciferase/Renilla.

Real-time PCR

Total RNA was isolated with TRIzol Reagent (Invitrogen) according to the manufacturer's instructions. The NTRK3 primers used were 5'-GTGAATGTGACGAGCGAAGA-3' and 5'-GGAGGGTAGTAGACAGTGAGAG $-3^{\prime}$. RT-PCR was performed with a CFX96 ${ }^{\text {TM }}$ Real-Time PCR Detection System (Bio-Rad Laboratories Co., Hercules, CA, USA). Relative mRNA levels were calculated and standardized with the GAPDH internal control using the $2^{-\triangle \triangle C T}$ method. The extraction of miRNAs was performed using the miRVana extraction kit (Ambion, Austin, TX, USA). The reverse transcription process was performed using the Taqman miRNA Reverse Transcription Kit and specific let-7i-5p primers (ThermoFisher, CA, USA). All results were normalized to $U 6$ levels and calculated using the $2^{-\triangle \Delta C T}$ method (Applied Biosystems, Inc. Foster City, $\mathrm{CA})$.

MiRNA mimics, inhibitors, overexpression plasmid, and small interfering RNA transfection

The expression of let-7i was regulated using chemically synthesized mimics or inhibitors, which were designed and purchased from Ribobio (Ribobio Co., Ltd., Guangdong, China). The sequences of small interfering RNA (siRNA) duplex for NTRK3 were also purchased from Ribobio company (Guangdong, China). Cells were divided into five groups transfected with miRNA mimics, miRNA inhibitors, RNAi, and overexpression plasmid separately. NTRK3 siRNA and overexpression plasmid were transfected using Lipofectamine $3000^{\text {TM }}$ following the manufacturer's instructions.

Western blot analysis

Western blot analysis was used to demonstrate the modulation of let-7i-5p on NTRK3. The transfected cells were lysed and homogenized in RIPA buffer. Approximately $20 \mu \mathrm{g}$ protein was loaded onto $10 \%$ polyacrylamide gels and separated on the BioRad mini gel system (Hercules, CA). Primary antibodies of NTRK3 (gtx83316, GeneTex) and GAPDH (gtx100118, GeneTex) were incubated overnight at $4{ }^{\circ} \mathrm{C}$. The HRP-labeled secondary antibody (1:5000; PIERCE, Rockford, USA) was incubated for $2 \mathrm{~h}$ at room temperature. Bands were visualized with colorimetry using a chemiluminescent imaging analyzer (Tanon 5200, Tanon Science \& Technology Co., Ltd., Shanghai, China).

Immunofluorescence staining

To evaluate NSC differentiation, neuronal differentiation-related markers were detected by immunofluorescence staining. Cells or frozen sections of Sprague-Dawley rat spinal cord tissues were fixed with $4 \%$ paraformaldehyde and then pretreated with $0.8 \%$ Triton X-100 to increase the permeability 
of the cell membrane. The primary antibodies anti-Tuj1, anti-MAP2, and anti-GFAP (1:1000, Millipore, Temecula, CA, USA) were applied overnight at $4{ }^{\circ} \mathrm{C}$. This was followed by three washes with phosphatebuffered saline and then incubation with secondary antibodies at room temperature for $2 \mathrm{~h}$. An inverted fluorescent microscope (Zeiss 200, Carl Zeiss, Jena, Germany) was used to capture the fluorescent images of 2D cultured cells. The fluorescent images of 3D cultured cells and frozen sections were observed by a scanning laser confocal fluorescence microscope (Leica TCS SP5, Leica Microsystems, Inc., Germany).

Cell migration assay

The cell migration assay for NSCs was performed in a 24-well plate Boyden chamber (Corning-3422, Corning Costar Corp, Cambridge, MA, USA). After culturing for 12 days, the cells were harvested from the collagen scaffold by collagenase type I (Invitrogen, Carlsbad, CA). The single cell suspension $\left(1 \times 10^{4}\right.$ cells) was placed in the upper chamber that had been coated with poly-D-lysine. The proliferation medium in the lower chamber contained $0.5 \mu \mathrm{g} / \mathrm{mL}$ SDF-1 (R\&D systems, MA, USA). Following incubation for $24 \mathrm{~h}$ at $37^{\circ} \mathrm{C}$, the cells on the upper surface of the membrane were scrubbed by cotton swab. The bottom side of the membrane was fixed with $4 \%$ paraformaldehyde and stained with hematoxylin and eosin. The total number of migrated cells were counted using a light microscope. The stained cells were calculated from three randomly chosen fields at a magnification of $20 x$.

Statistical analyses

All results were statistically analyzed and presented as mean \pm SD from at least three independent experiments. The Student's $t$-test and Factorial analysis of variance (ANOVA) were analyzed with SPSS (SPSS version 13.0; SPSS Inc., Chicago, IL, USA). Other analyses were performed using Prism 3.0 (Graphpad Software Inc., San Diego, CA, USA).

\section{Results}

\section{The morphology and good cytocompatibility of the collagen sponge scaffold}

SEM revealed that the collagen sponge scaffold possesses a rich internal porous structure and good porosity (Fig. 1A). The porous structure contributes to the transport of oxygen, nutrients, and waste. The average pore diameter was $40.69 \mu \mathrm{m}$ with good porosity. The 3D structure allows the cells to stretch out in all directions. Good NSC adhesion and growth were observed on the surface and in the pores of the collagen sponge scaffold (Fig. 1B). FDA staining also revealed that the cells attached and spread well on the collagen sponge scaffold (Fig. 1C). These results indicate that the collagen sponge scaffold represents an excellent alternative to $2 \mathrm{D}$ culturing of NSCs.

\section{The effect of the RCCS on the cellular properties of NSCs}


Previous studies have reported that microgravity can alter cellular properties. Here, we evaluated the effect of simulated microgravity on the cellular properties of NSCs. Immunofluorescence staining (Fig. $2 \mathrm{~A}$ ) and the migration assay (Fig. 2B) indicated, compared with the control group, increased neuronal differentiation and migration and decreased GFAP expression of 3D cultured NSCs in the RCCS. Immunofluorescence staining revealed that the NSCs cultured in the RCCS bioreactor exhibited enhanced neuronal differentiation and suppression of astrocytic differentiation in NT-3-containing differentiation medium.

Both real-time PCR and RNA sequencing showed increased NTRK3 expression in the RCCS group compared with the control group (Fig. 3A). Thus, we further evaluated the effect of simulated microgravity on NSCs in the presence of NT-3. There is a large amount of evidence that NTRK3 is widely expressed in the brain and lumbar spinal cord and has a high affinity for NT-3[27-29]. In the RCCS, neuronal differentiation and migratory ability were increased, whereas the differentiation ability of astrocytes was decreased in NT-3-containing versus traditional medium. Taken together, these results indicate that the RCCS contributes to the neuronal differentiation and migration of NSCs, especially in differentiation medium containing NT-3.

\section{The direct regulatory role between let-7i-5p and NTRK3}

The analysis of the mRNA sequencing data identified some differentially expressed mRNAs in NSCs between the traditional and RCCS cell culture systems. This altered mRNA expression may elucidate the mechanisms underlying the effects of the RCCS on 3D cultured NSCs. Using the miRNA target gene pairs predicted by TargetScan, we constructed the regulatory network between expressed mRNAs and miRNAs predicted to be involved in regulating them. Among the regulatory network, the regulatory relationship between NTRK3 and let-7i-5p caught our attention. The bioinformatics analysis indicated that the upregulation of NTRK3 may be regulated by the downregulation of let-7i-5p (Fig. 3B). To prove the accuracy of the RNA sequence data, we performed quantitative real-time PCR (Q-PCR). Consistent with the RNA sequencing data, Q-PCR showed that the relative expression of NTRK3 increased by four-fold in the RCCS group, whereas the relative expression of let-7i-5p decreased by nearly $20 \%$ (Fig. 3A).

Considering that the bioinformatics analysis indicated that the 3'UTR of the NTRK3 gene contained the let-7i-5p binding sites, we performed the luciferase report assay to test the interaction between them. Luc activity was the lowest in the wild plasmid (WT, the vector containing wild-type 3'-UTR of NTRK3) combined with the let-7i-5p mimics in HeLa cells, whereas significantly upregulated in the wild plasmid combined with let-7i-5p inhibitors. When the binding site in the wild plasmid was mutated (MUT, the vector containing mutant 3 '-UTR of NTRK3), no significant alteration was detected when combined with let-7i-5p mimics (Fig. 3C). These results suggest that the NTRK3 binding site is required for miRNA binding and activity. The Western blot analysis further validated the regulatory role between let-7i-5p and NTRK3 in NSCs. Compared with the control group, the NTRK3 protein level was decreased in the let-7i-5p inhibitor group and increased in the let-7i-5p mimic group (Fig. 3D). 
To further validate the regulatory roles of let-7i-5p and NTRK3, we performed immunofluorescence staining and trans-well migration assay to compare the differentiation (Fig. 4) and migratory (Fig. 5) ability of NSCs between six groups: ctr, let-7i-5p mimics, let-7i-5p inhibitors, NTRK3 siRNA, NTRK3 overexpression, and let-7i-5p inhibitor+NTRK3 siRNA. NT-3-containing medium was used in all groups. Consistent with NSCs transfected with let-7i-inhibitor, the neuronal differentiation and migratory ability of NSCs transfected with NTRK3 overexpression plasmid was enhanced, accompanied with a decreased ability for astrocyte differentiation. Meanwhile, neuronal differentiation and migratory ability were poor in the let-7i-5p-mimics and NTRK3 siRNA groups. Additionally, the positive effect of the let-7i-5p-inhibitor on neuronal differentiation and migration can be reversed when combined with NTRK3 siRNA. When NTRK3 was knocked down by siRNA, the boost from the let-7i-5p-inhibitor was undermined. As such, these results suggest that NTRK3 is a target gene of let-7i-5p and plays a certain role in regulating neuronal differentiation and migration, especially when exposed to exogenous NT-3. Overexpression or knockdown of let-7i-5p in NSCs further indicated that let-7i plays an inhibitory role on the expression of its target gene NTRK3. Taken together, we have demonstrated for the first time that microRNA let-7i-5p is a negative regulator of NTRK3 in NSCs.

\section{The migratory ability of implanted NSCs in animal models of spinal cord injury}

To further assess the migratory ability of 3D cultured NSCs in different groups, we then implanted collagen sponge scaffold with cells at the injury site in rats with spinal cord injury (Fig. 6A). Immunofluorescent staining indicated that the implanted cells remained within the implanted injury site in both the static and static+NT-3 groups 2 weeks after implantation (Fig. 6B). Conversely, a small fraction of cells in the RCCS and RCCS+NT-3 groups migrated from the implanted lesion site to the injury site. Compared with the RCCS group, the RCCS+NT-3 group exhibited more NSC migration and with longer distances (Fig. 6C). Consistent with the cell experiments, the in vivo animal tests further demonstrated that 3D cultured NSCs in the RCCS environment possess better migratory ability than that of other groups, especially in the presence of NT-3. The migratory ability of NSCs in the RCCS+NT-3 group was enhanced compared with that of other groups at the injury site, indicating that NT-3 may improve the therapeutic effects of transplanted NSCs.

\section{Discussion}

Due to the lack of regeneration in the CNS, spontaneous repair is restricted after injury or degeneration. The transplantation of NSCs has a promising future both for stem cell therapy and scientific research. Currently, cell therapy is a hotspot in neuroscience research. Multiple preclinical trials have convincingly demonstrated that NSC transplantation promotes a positive outcome (i.e, neurogenesis) in rodent models, indicating a novel approach for the treatment of various CNS diseases[30-32]. Nevertheless, the promising application of NSC transplantation remains elusive, since many grafted cells differentiate into glial cells, which form scars instead of repairing the damaged structure and function $[9,10]$. Glial scarring is the main obstacle to nerve regeneration $[33,34]$ and warrants further research. Acquiring suitable graft 
cells is the critical element that could ensure treatment efficacy. Currently, more and more researchers are focused on improving the bioprocessing method to produce appropriate cells for tissue engineering.

The culturing environment is one of the most important pivotal factors that influence cell function and its potential therapeutic effects following transplantation. Our previous findings demonstrated that NSCs tend to differentiate into neurons and that glial differentiation is inhibited during spaceflight[11]. Considering that microgravity is a key factor in space, we have here investigated the effects of microgravity on NSC function using a RCCS bioreactor, a simulated microgravity device, which was designed by NASA (US National Aeronautics and Space Administration) [35]. The RCCS enables cell culture experiments to be manipulated in simulated microgravity environments as well as during space flight, the application of microgravity environments in the fields of stem cell research and therapy is increasing. A recent study showed the changes of rostral-caudal neural patterning genes and cortical markers in human embryonic stem cell (hESC)-derived neural organoids cultured in microgravity conditions compared with standard conditions[36]. Additionally, emerging evidence indicates that microgravity affects not only cell differentiation but also the therapeutic effect of transplanted cells[15, 37]. Several studies have demonstrated that microgravity contributes to providing seeding cells for cell therapy and enhancing the therapeutic effect of stem cell transplantation[38-40]. Most notably, the application of simulated microgravity for stem cell-based therapy in the treatment of CNS diseases has also been reported, indicating that microgravity could improve therapeutic effects of mesenchymal stem cell (MSCs) for CNS diseases[41]. Taken together, evidence indicates that the RCCS can be superior to static culture conditions.

NSCs cultivated in the RCCS tend to aggregate and form big neurospheres. To prevent NSC aggregation, we seeded the NSCs onto collagen sponge scaffolds, which provide a 3-D environment for cells to attach and stretch out in all directions. Such 3-D cell culturing is a novel technology that is being widely applied in tissue engineering[ $[42,43]$. The collagen sponge scaffold we used in this study had been proven to be biocompatible and biodegradable in our previous studies $[19,44]$. Our data indicate that NSCs grow and attach well on the scaffold, suggesting that this scaffold may be the optimal cell carrier for nerve tissue engineering and may provide a valuable platform to produce seeding cells. Furthermore, collagen sponge scaffolds with attached NSCs are convenient to graft during cell transplantation for cell therapy.

In view of the advantages of the RCCS and 3D collagen sponge scaffold cell culture system, we aimed to evaluate whether the combination of these systems could represent a good tissue engineering approach to provide suitable NSCs for future cellular therapies in neurology. Both RNA sequencing and RT-PCR analysis revealed elevated NTRK3 expression under microgravity conditions in the RCCS. Furthermore, we demonstrated that NTRK3 is the target gene of let-7i-5p. A previous study demonstrated that inhibiting let$7 i-5 p$ enhances neuroprotection and facilitates functional recovery following stroke[45]; however, the functions of let-7i-5p in NSCs remain unclear. By performing luciferase assay and Western blot, we validated a direct regulatory role between NTRK3 and let-7i-5p that was predicted by our bioinformatics analysis. We also demonstrated that the positive effect of let-7i-5p-inhibitor on neuronal differentiation and migration in NT-3-containing differentiation medium can be reversed by adding NTRK3 siRNA. 
It had been reported that NTRK3 overexpression may increase the capacity for neuronal differentiation[46]. It is well known that the neurotrophin NT-3 plays an important role in regulating neuronal differentiation and nerve regeneration. Nevertheless, neural precursor cells do not express sufficient levels of Trk receptors; this limits the positive effect of NT-3 on nerve regeneration. To enable the over-expressed NTRK3 to exert the biggest impact, a sufficient concentration of NT-3 in the differentiation medium is crucial. Our findings demonstrated that NTRK3-overexpressing cells promote migration and early neuronal differentiation and also suppress astrocytic differentiation. Compared with gene modification, this method is simpler, faster, and more easily adaptable to obtaining NSCs with high NTRK3 expression. Therefore, our method that combines the 3D collagen sponge-based cell culture system with the RCCS bioreactor is a promising strategy for tissue engineering and is expected to be used in future clinical studies.

\section{Conclusions}

In summary, our results indicate that 3D cultured NSCs in RCCS bioreactors exhibit better neuronal differentiation and migratory ability than traditional static cultures. This is important for cell transplantation, as excellent neuronal differentiation and migratory ability increase treatment efficacy. Our initial findings suggest that employing a RCCS bioreactor in combination with NT-3 is a useful strategy to provide effective NSCs for stem cell therapy. The combination of the RCCS bioreactor and NT3-containing medium may provide an attractive solution for therapeutic cell therapy demands in the future.

\section{Abbreviations}

RCCS Rotary Cell Culture System

NTRK3 neurotrophic receptor tyrosine kinase 3

NSCs Neural stem cells

NT-3 Recombinant Neurotrophin 3

3D three-dimensional

CNS central nervous system

2D two-dimensional

SD rats Sprague-Dawley rats

GFP Green fluorescent protein

SEM scanning electron microscope 
FDA Fluorescein diacetate

UTR Untranslated Region

Q-PCR quantitative real-time PCR

siRNAs Small interfering RNAs

Tuj1 Neuronal Class III $\beta$-Tubulin

Map2 microtubule associated protein 2

GFAP glial fibrillary acidic protein

ANOVA Analysis of Variance

$\mathrm{SCl} \quad$ Spinal cord injury

NASA US National Aeronautics and Space Administration

\section{Declarations}

\section{Acknowledgements}

We thank Eva Lasic, PhD, from Edanz (https://en-author-services.edanz.com/) for editing a draft of this manuscript.

\section{Funding}

This work was supported by the National Research Institute for National Key R\&D Program of China (2016YFC1000806/2016YFC1000801/2016YFC1000802), the National Natural Science Foundation of China (U1738109,82071403), and the Strategic Priority Research Program of the Chinese Academy of Sciences (XDA16040600/ XDA16040700).

\section{Availability of data and materials}

The datasets supporting the conclusions of this article are included within the article

\section{Ethics approval and consent to participate}

All institutional and national guidelines for the care and use of laboratory animals were followed. This article does not contain any studies with human subjects performed by any of the authors.

\section{Competing interests}

The authors declare that they have no competing interests. 


\section{Authors' contributions}

$Y C$ has substantial contributions to the conception and design; acquisition, analysis, and interpretation of the data; and manuscript writing. YYY, XB have substantial contributions to the animal studies. JH, YNZ and YLZ contributed to the collagen sponge scaffold preparation. BC, ZFX, YS and WWX have substantial contributions to the cell culture. HWS has substantial contributions to the analysis of microarray data. JWD and XM have substantial contributions to the conception and design, financial support, and analysis and interpretation of the data. All authors read and approved the final manuscript.

\section{Consent for publication}

Not applicable.

\section{References}

[1] Bernstock JD, L Peruzzotti-Jametti, D Ye, FA Gessler, D Maric, N Vicario, et al., Neural stem cell transplantation in ischemic stroke: A role for preconditioning and cellular engineering. J Cereb Blood Flow Metab, 2017; 37: 2314-2319.

[2] Gonzalez R, MH Hamblin, and JP Lee, Neural Stem Cell Transplantation and CNS Diseases. CNS Neurol Disord Drug Targets, 2016; 15: 881-886.

[3] Qiao S, Y Liu, F Han, M Guo, X Hou, K Ye, et al., An Intelligent Neural Stem Cell Delivery System for Neurodegenerative Diseases Treatment. Adv Healthc Mater, 2018; 7: e1800080.

[4] Garitaonandia I, R Gonzalez, G Sherman, A Semechkin, A Evans, and R Kern, Novel Approach to Stem Cell Therapy in Parkinson's Disease. Stem Cells Dev, 2018; 27: 951-957.

[5] McGinley LM, ON Kashlan, ES Bruno, KS Chen, JM Hayes, SR Kashlan, et al., Human neural stem cell transplantation improves cognition in a murine model of Alzheimer's disease. Sci Rep, 2018; 8: 14776.

[6] Weston NM and D Sun, The Potential of Stem Cells in Treatment of Traumatic Brain Injury. Curr Neurol Neurosci Rep, 2018; 18: 1.

[7] Xiong LL, Y Hu, P Zhang, Z Zhang, LH Li, GD Gao, et al., Neural Stem Cell Transplantation Promotes Functional Recovery from Traumatic Brain Injury via Brain Derived Neurotrophic FactorMediated Neuroplasticity. Mol Neurobiol, 2018; 55: 2696-2711.

[8] Zhu Y, N Uezono, T Yasui, and K Nakashima, Neural stem cell therapy aiming at better functional recovery after spinal cord injury. Dev Dyn, 2018; 247: 75-84.

[9] Enzmann GU, RL Benton, JF Talbott, Q Cao, and SR Whittemore, Functional considerations of stem cell transplantation therapy for spinal cord repair. J Neurotrauma, 2006; 23: 479-95. 
[10] Hofstetter CP, NA Holmstrom, JA Lilja, P Schweinhardt, J Hao, C Spenger, et al., Allodynia limits the usefulness of intraspinal neural stem cell grafts; directed differentiation improves outcome. Nat Neurosci, 2005; 8: 346-53.

[11] Cui Y, J Han, Z Xiao, Y Qi, Y Zhao, B Chen, et al., Systematic Analysis of mRNA and miRNA Expression of 3D-Cultured Neural Stem Cells (NSCs) in Spaceflight. Front Cell Neurosci, 2017; 11: 434.

[12] Grimm D, M Egli, M Kruger, S Riwaldt, TJ Corydon, S Kopp, et al., Tissue Engineering Under Microgravity Conditions-Use of Stem Cells and Specialized Cells. Stem Cells Dev, 2018; 27: 787-804.

[13] Zhang C, L Li, J Chen, and J Wang, Behavior of stem cells under outer-space microgravity and ground-based microgravity simulation. Cell Biol Int, 2015; 39: 647-56.

[14] Li BB, ZY Chen, N Jiang, S Guo, JQ Yang, SB Chai, et al., Simulated microgravity significantly altered metabolism in epidermal stem cells. In Vitro Cell Dev Biol Anim, 2020; 56: 200-212.

[15] Imura T, T Otsuka, Y Kawahara, and L Yuge, "Microgravity" as a unique and useful stem cell culture environment for cell-based therapy. Regen Ther, 2019; 12: 2-5.

[16] Morabito C, N Steimberg, G Mazzoleni, S Guarnieri, G Fano-Illic, and MA Mariggio, RCCS bioreactor-based modelled microgravity induces significant changes on in vitro 3D neuroglial cell cultures. Biomed Res Int, 2015; 2015: 754283.

[17] Cui Y, Z Xiao, T Chen, J Wei, L Chen, L Liu, et al., The miR-7 identified from collagen biomaterialbased three-dimensional cultured cells regulates neural stem cell differentiation. Stem Cells Dev, 2014; 23: 393-405.

[18] Han S, Y Zhao, Z Xiao, J Han, B Chen, L Chen, et al., The three-dimensional collagen scaffold improves the stemness of rat bone marrow mesenchymal stem cells. J Genet Genomics, 2012; 39: 63341.

[19] Han J, Z Xiao, L Chen, B Chen, X Li, S Han, et al., Maintenance of the self-renewal properties of neural progenitor cells cultured in three-dimensional collagen scaffolds by the REDD1-mTOR signal pathway. Biomaterials, 2013; 34: 1921-8.

[20] Han Q, JD Ordaz, NK Liu, Z Richardson, W Wu, Y Xia, et al., Descending motor circuitry required for NT-3 mediated locomotor recovery after spinal cord injury in mice. Nat Commun, 2019; 10: 5815.

[21] Yang JT, TH Lee, HH Weng, CN Chang, WC Chen, WC Cheng, et al., Dexamethasone enhances NT3 expression in rat hippocampus after traumatic brain injury. Exp Neurol, 2005; 192: 437-43.

[22] Szobota S, PD Mathur, S Siegel, K Black, HU Saragovi, and AC Foster, BDNF, NT-3 and Trk receptor agonist monoclonal antibodies promote neuron survival, neurite extension, and synapse restoration in rat cochlea ex vivo models relevant for hidden hearing loss. PLoS One, 2019; 14: e0224022. 
[23] Akyol O, P Sherchan, G Yilmaz, C Reis, WM Ho, Y Wang, et al., Neurotrophin-3 provides neuroprotection via TrkC receptor dependent pErk5 activation in a rat surgical brain injury model. Exp Neurol, 2018; 307: 82-89.

[24] Edalat $\mathrm{H}, \mathrm{Z}$ Hajebrahimi, $V$ Pirhajati, M Tavallaei, M Movahedin, and SJ Mowla, Exogenous Expression of Nt-3 and TrkC Genes in Bone Marrow Stromal Cells Elevated the Survival Rate of the Cells in the Course of Neural Differentiation. Cell Mol Neurobiol, 2017; 37: 1187-1194.

[25] Han J, B Wang, Z Xiao, Y Gao, Y Zhao, J Zhang, et al., Mammalian target of rapamycin (mTOR) is involved in the neuronal differentiation of neural progenitors induced by insulin. Mol Cell Neurosci, 2008; 39: 118-24.

[26] Jones K, DW Kim, JS Park, and CH Khang, Live-cell fluorescence imaging to investigate the dynamics of plant cell death during infection by the rice blast fungus Magnaporthe oryzae. BMC Plant Biol, 2016; 16: 69.

[27] Uriguen L, D Arteta, R Diez-Alarcia, M Ferrer-Alcon, A Diaz, A Pazos, et al., Gene expression patterns in brain cortex of three different animal models of depression. Genes Brain Behav, 2008; 7: 64958.

[28] Tessarollo L, KS Vogel, ME Palko, SW Reid, and LF Parada, Targeted mutation in the neurotrophin-3 gene results in loss of muscle sensory neurons. Proc Natl Acad Sci U S A, 1994; 91: 118448.

[29] Hsiao SJ, A Zehir, AN Sireci, and DL Aisner, Detection of Tumor NTRK Gene Fusions to Identify Patients Who May Benefit from Tyrosine Kinase (TRK) Inhibitor Therapy. J Mol Diagn, 2019; 21: 553-571.

[30] Goldman SA, S Schanz, and MS Windrem, Stem cell-based strategies for treating pediatric disorders of myelin. Hum Mol Genet, 2008; 17: R76-83.

[31] Tuazon JP, V Castelli, JY Lee, GB Desideri, L Stuppia, AM Cimini, et al., Neural Stem Cells. Adv Exp Med Biol, 2019; 1201: 79-91.

[32] Stenudd M, H Sabelstrom, and J Frisen, Role of endogenous neural stem cells in spinal cord injury and repair. JAMA Neurol, 2015; 72: 235-7.

[33] Chen X and W Zhu, A Mathematical Model of Regenerative Axon Growing along Glial Scar after Spinal Cord Injury. Comput Math Methods Med, 2016; 2016: 3030454.

[34] Zhang JX, WW Hu, and Z Liu, Recent advances in treatment of glial scar-induced inhibition of nerve regeneration. Zhejiang Da Xue Xue Bao Yi Xue Ban, 2009; 38: 639-43.

[35] Begley CM and SJ Kleis, The fluid dynamic and shear environment in the NASA/JSC rotating-wall perfused-vessel bioreactor. Biotechnol Bioeng, 2000; 70: 32-40. 
[36] Mattei C, A Alshawaf, G D'Abaco, B Nayagam, and M Dottori, Generation of Neural Organoids from Human Embryonic Stem Cells Using the Rotary Cell Culture System: Effects of Microgravity on Neural Progenitor Cell Fate. Stem Cells Dev, 2018; 27: 848-857.

[37] Kurose T, S Takahashi, T Otsuka, K Nakagawa, T Imura, T Sueda, et al., Simulated microgravitycultured mesenchymal stem cells improve recovery following spinal cord ischemia in rats. Stem Cell Res, 2019; 41: 101601.

[38] Yin $\mathrm{H}, \mathrm{Y}$ Wang, $X$ Sun, G Cui, Z Sun, $\mathrm{P}$ Chen, et al., Functional tissue-engineered microtissue derived from cartilage extracellular matrix for articular cartilage regeneration. Acta Biomater, 2018; 77: 127-141.

[39] Pao SI, KH Chien, HT Lin, MC Tai, JT Chen, and CM Liang, Effect of microgravity on the mesenchymal stem cell characteristics of limbal fibroblasts. J Chin Med Assoc, 2017; 80: 595-607.

[40] Hou Y, C Song, WJ Xie, Z Wei, RP Huang, W Liu, et al., Excellent effect of three-dimensional culture condition on pancreatic islets. Diabetes Res Clin Pract, 2009; 86: 11-5.

[41] Imura T, K Nakagawa, Y Kawahara, and L Yuge, Stem Cell Culture in Microgravity and Its Application in Cell-Based Therapy. Stem Cells Dev, 2018; 27: 1298-1302.

[42] Chen $\mathrm{Q}$ and $\mathrm{Y}$ Wang, The application of three-dimensional cell culture in clinical medicine. Biotechnol Lett, 2020; 42: 2071-2082.

[43] Zhang W, A Zhuang, P Gu, H Zhou, and X Fan, A review of the three-dimensional cell culture technique: Approaches, advantages and applications. Curr Stem Cell Res Ther, 2016; 11: 370-80.

[44] Zou Y, D Ma, H Shen, Y Zhao, B Xu, Y Fan, et al., Aligned collagen scaffold combination with human spinal cord-derived neural stem cells to improve spinal cord injury repair. Biomater Sci, 2020; 8: 5145-5156.

[45] Nguyen T, C Su, and M Singh, Let-7i inhibition enhances progesterone-induced functional recovery in a mouse model of ischemia. Proc Natl Acad Sci U S A, 2018; 115: E9668-E9677.

[46] Wang JM, YS Zeng, JL Wu, Y Li, and YD Teng, Cograft of neural stem cells and schwann cells overexpressing TrkC and neurotrophin-3 respectively after rat spinal cord transection. Biomaterials, 2011; 32: 7454-68.

\section{Figures}


(A)

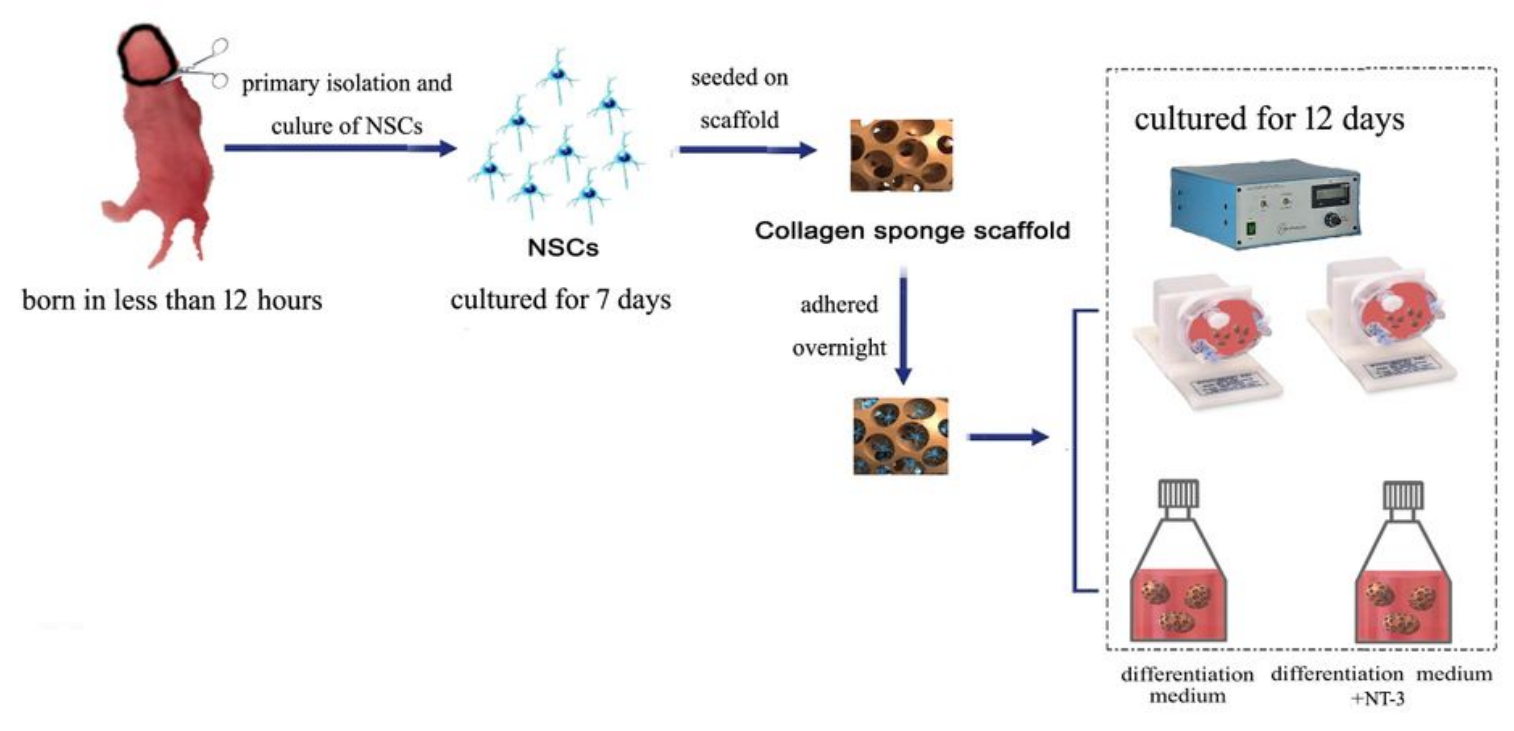

(B)
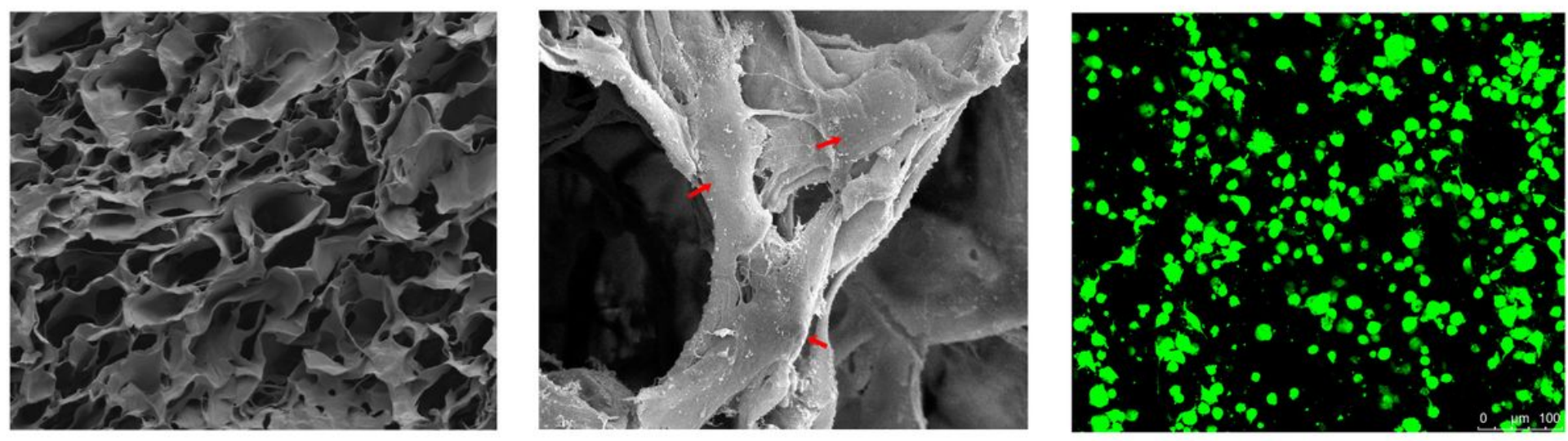

Figure 1

(A) A flowchart of the experiment. (B) Scanning electron microscopy images reveal the morphology of the collagen sponge scaffold (left) and 3D cultured neural stem cells (NSCs) (middle). The red arrow indicates a cell that attached to the scaffold. A representative cell image of fluorescein diacetate staining of the 3D cultured NSCs (right). 
(A)
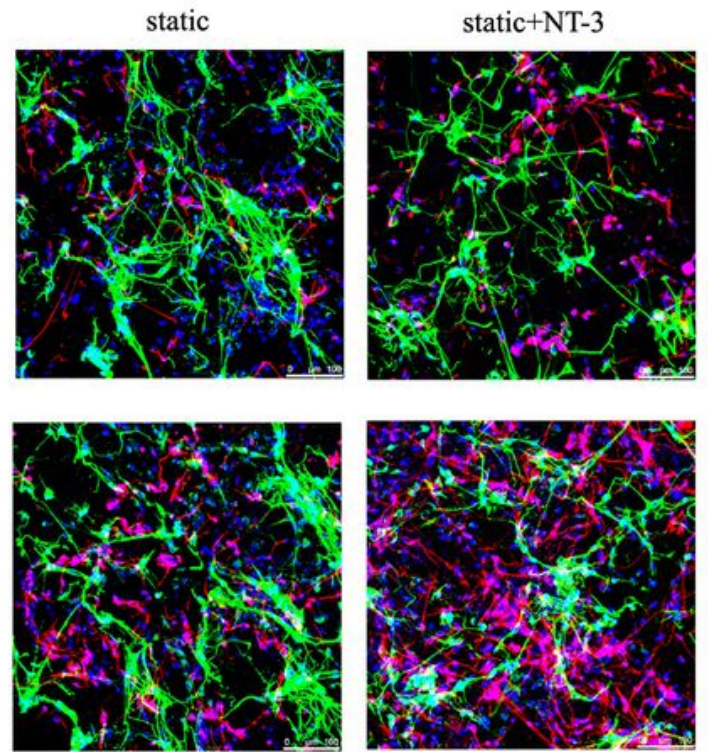

RCCS
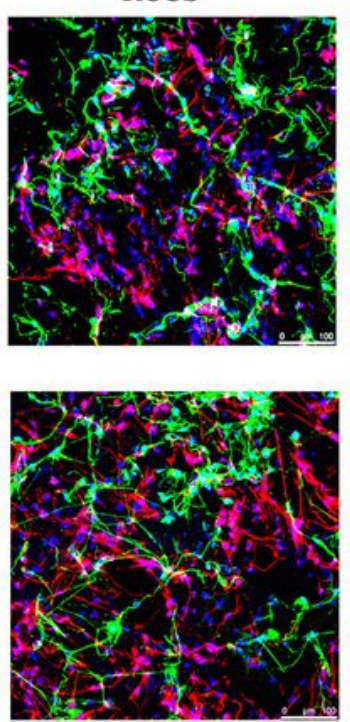

RCCS+NT-3
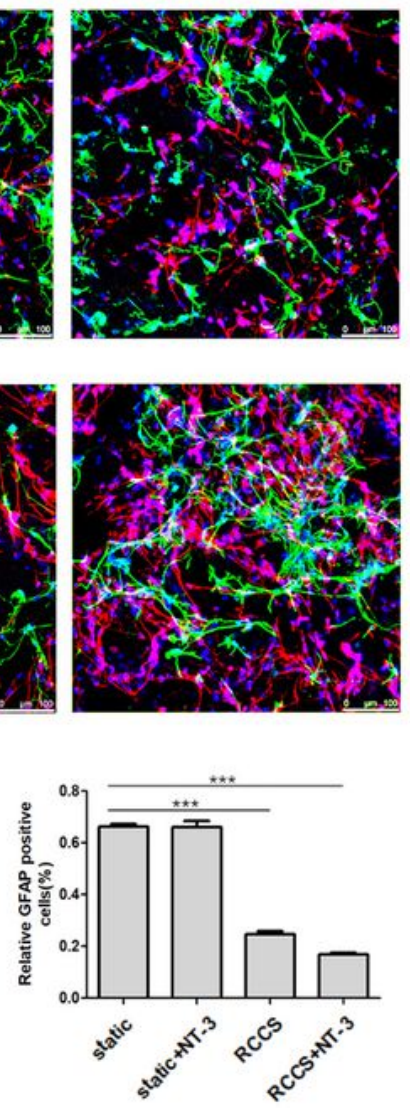

(B)
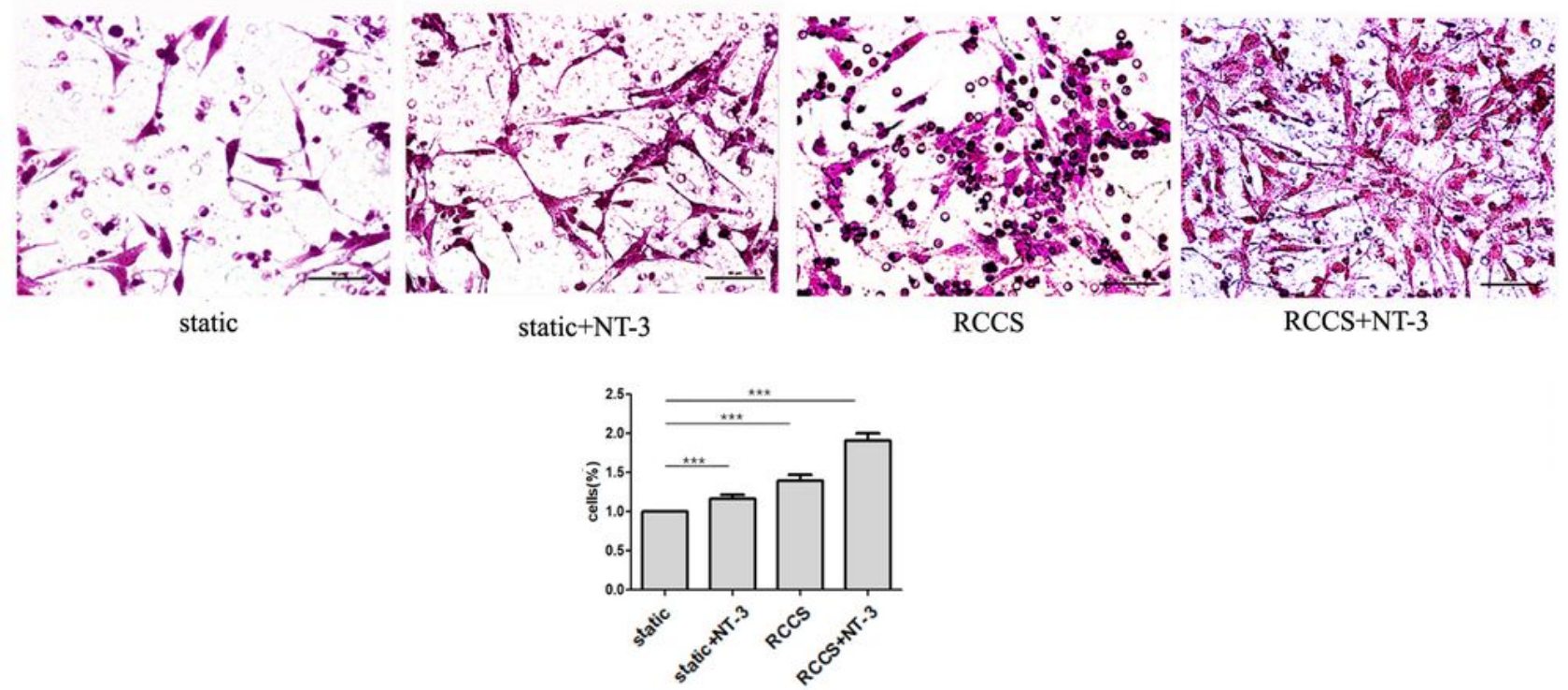

Figure 2

The differentiation and migratory ability of 3D cultured NSCs in all groups detected by immunofluorescence staining and transwell migration array. (A) Representative images of immunofluorescence double staining of neural differentiation related marker Tuj1, Map2 and astrocyte differentiation related marker GFAP acquired by a confocal laser scanning microscope using a $20 \times$ objective lens. The red signal represents Tuj1 and Map2 positive cells. The green signal represents GFAP- 
positive cells. Nuclei were counterstained using Hoechst 33342. Scale bars: $100 \mu \mathrm{m}$. (B) Images of migrated cells detected by the transwell migration array were captured by a microscope using a $40 \times$ objective lens. Scale bars: $50 \mu \mathrm{m}$. Both the neural differentiation and migratory ability of 3D cultured NSCs in the RCCS group were elevated compared with traditional static cultures, especially in NT-3containing culture medium. Data represent mean \pm S.D.; $n=3 ; * \star * p<0.001$.

(A)

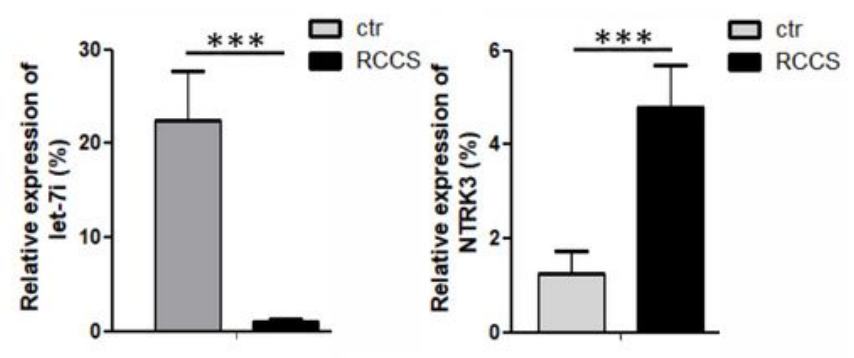

(B)

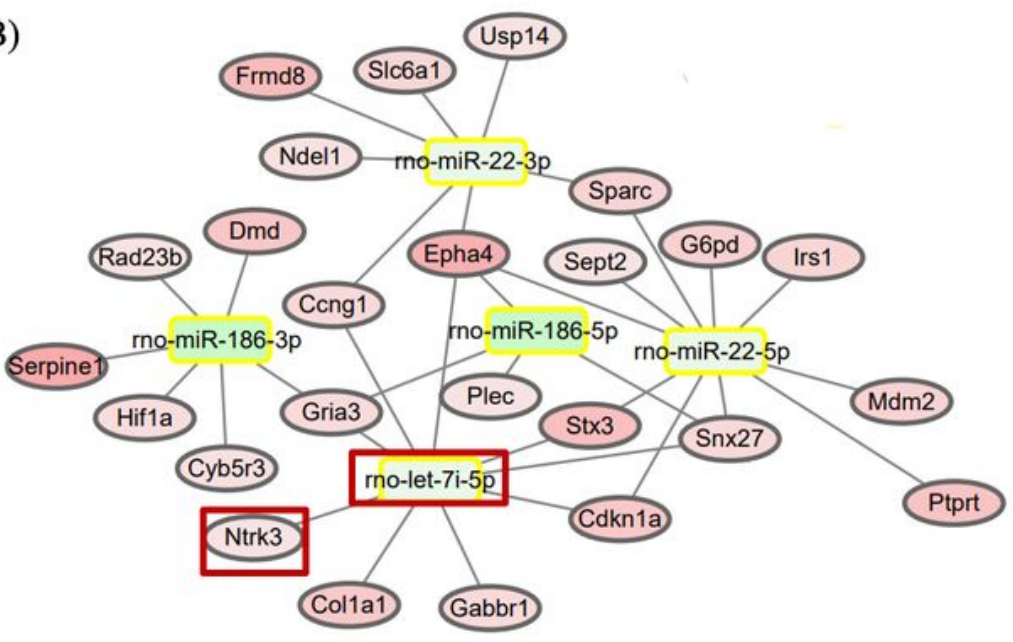

(C)
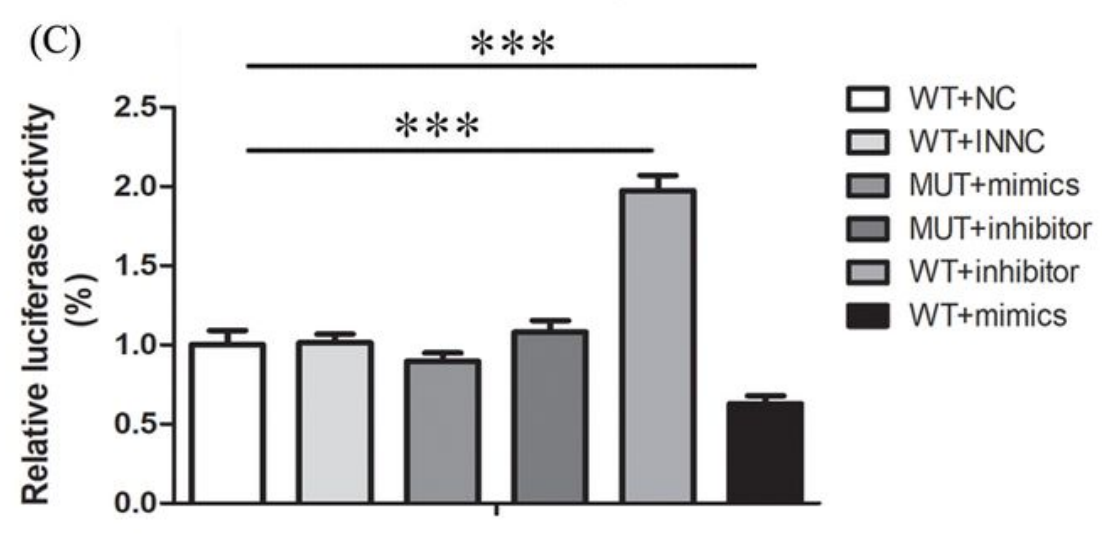

(D)

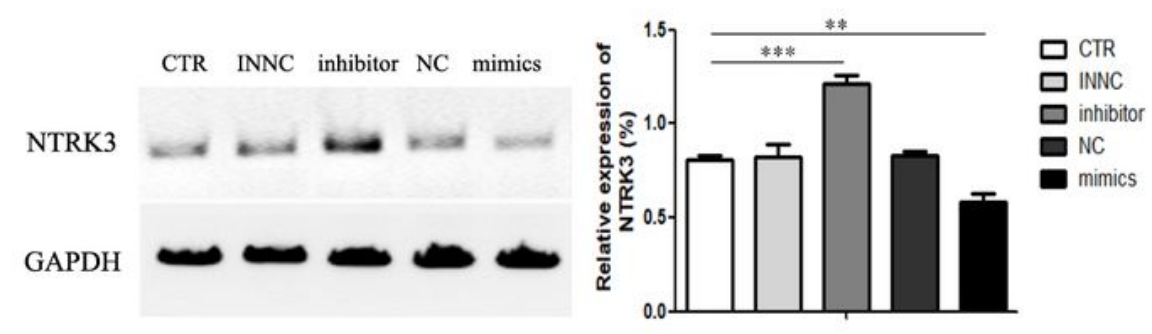

Figure 3 
The interaction between let-7i-5p and NTRK3. (A) Real-time PCR indicates let-7i-5p and NTRK3 expression in the RCCS and control groups. (B) Bioinformatics analysis predicted a regulatory role of let-7i-5p on NTRK3. (C) The luciferase reporter array indicated an interaction between 3'-UTR of NTRK3 and the seed sequence of let-7i-5p. Data are shown as mean \pm S.D. from three independent experiments. ${ }^{* \star} p<0.001$ versus corresponding WT+NC. WT: vector containing wild-type 3'-UTR of NTRK3; MUT: vector containing mutant 3'-UTR of NTRK3; INNC: inhibitor negative control; Inhibitor: let-7i-5p inhibitor; NC: negative control; mimics: let-7i-5p mimics. (D) Western blot indicated that NTRK3 protein levels are affected by let$7 \mathrm{i}-5 \mathrm{p}$ mimics and inhibitors. Data are shown as mean \pm S.D from three independent experiments. ${ }^{* \star} \mathrm{p}<0.01$ versus $C T R$, ${ }^{\star * *} \mathrm{p}<0.001$ versus $C T R$. 
(A)

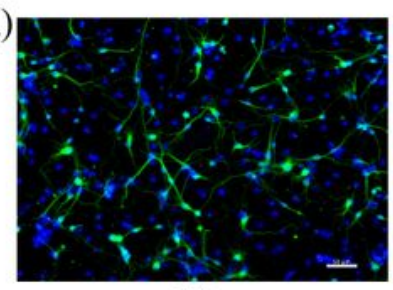

CTR

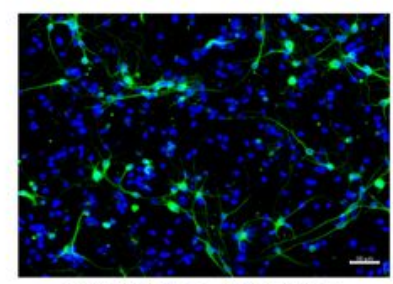

let-7i-inhibitor + NTRK3 siRNA

(B)

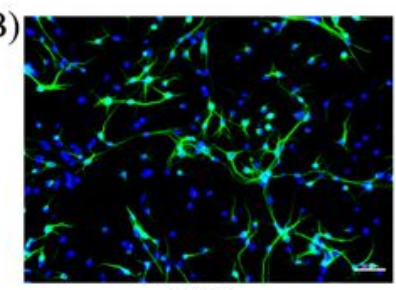

NTRK3

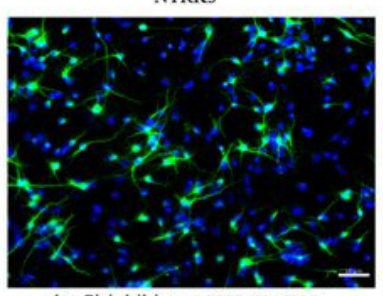

let-7i-inhibitor + NTRK3 siRNA

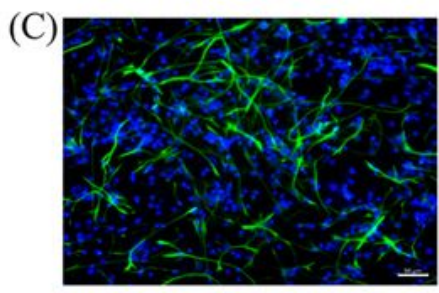

CTR

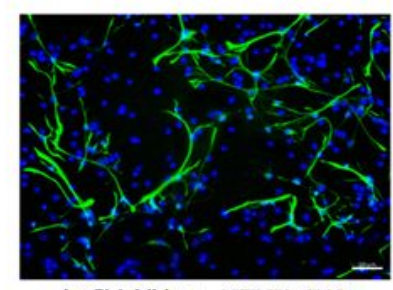

let-7i-inhibitor + NTRK3 siRNA

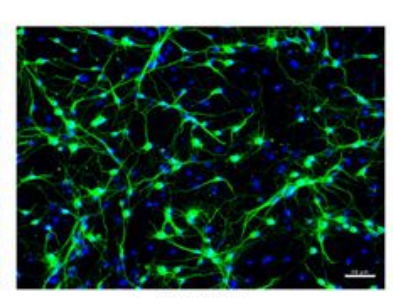

let-7i-inhibitor

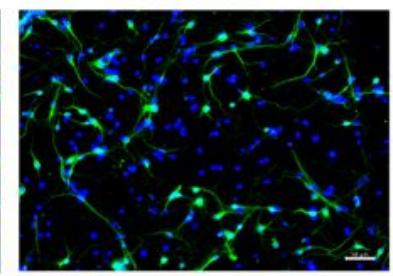

let-7i-mimics

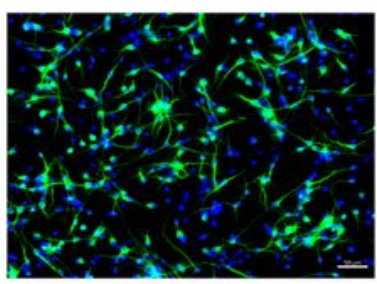

let-7i-inhibitor

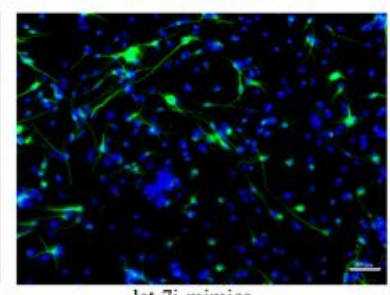

let-7i-mimics

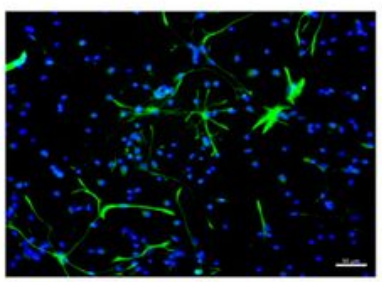

let-7i-inhibitor

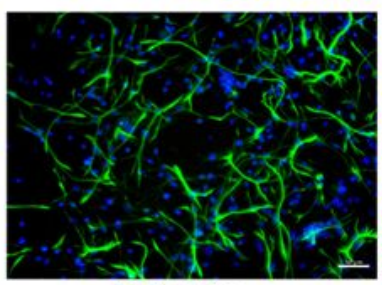

let-7i-mimics
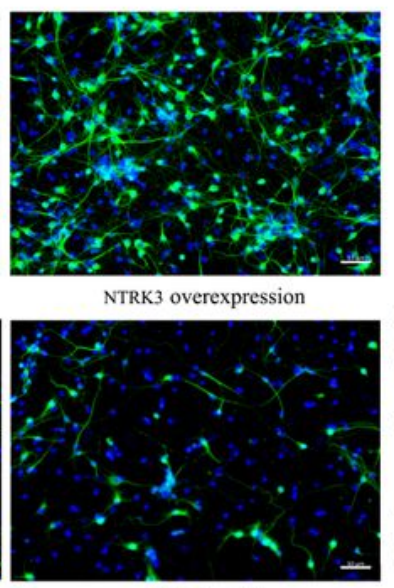

NTRK3 siRNA

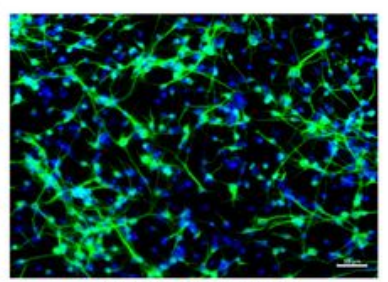

NTRK3 overexpression

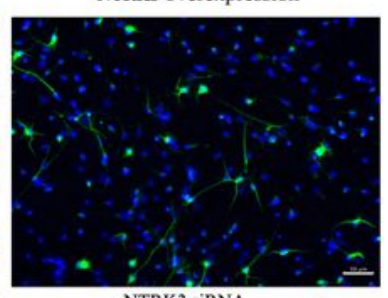

NTRK 3 siRNA

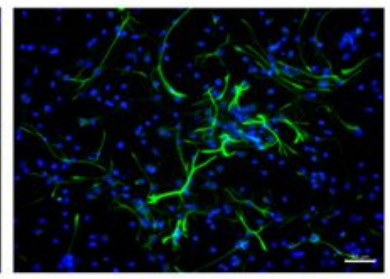

NTRK 3 overexpression

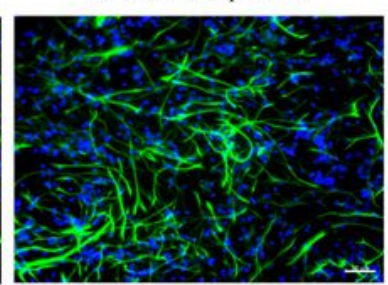

NTRK3 siRNA $\square$ CTR

$\square$ let7i-inhiibitor

$\square$ NTRK3 overexpression

$\square$ let7i-inhiibitor+siRNA

let7i mimics

$\square$ NTRK3 siRNA
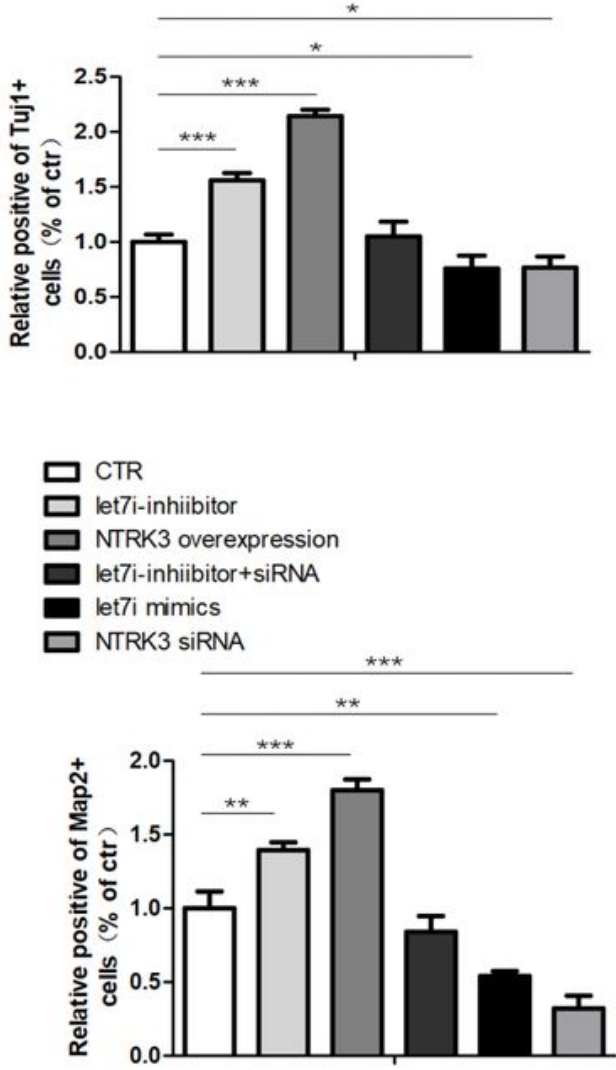

\section{$\square$ CTR
$\square$ let7i-inhiibitor \\ $\square$ NTRK3 overexpression \\ $\square$ let7i-inhiibitor+siRNA \\ let7i mimics \\ $\square$ NTRK3 siRNA}

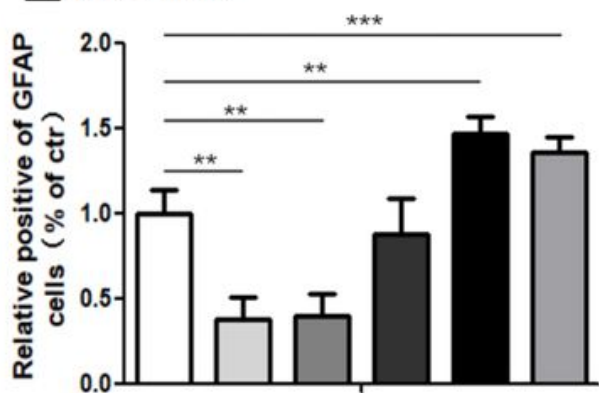

\section{Figure 4}

Immunofluorescence staining indicated an effect of let-7i-5p and NTRK3 on NSC differentiation when the differentiation medium contained NT-3. Representative images of (A) Tuj1-, (B) Map2-, and (C) GFAPpositive cells after transfection. Nuclei were counterstained using Hoechst 33342 . Scale bars: $50 \mu \mathrm{m}$. Data represent mean $\pm S$.D. from three independent experiments. ${ }^{*} P<0.05, * \star P<0.01, * \star \star P<0.001$. 

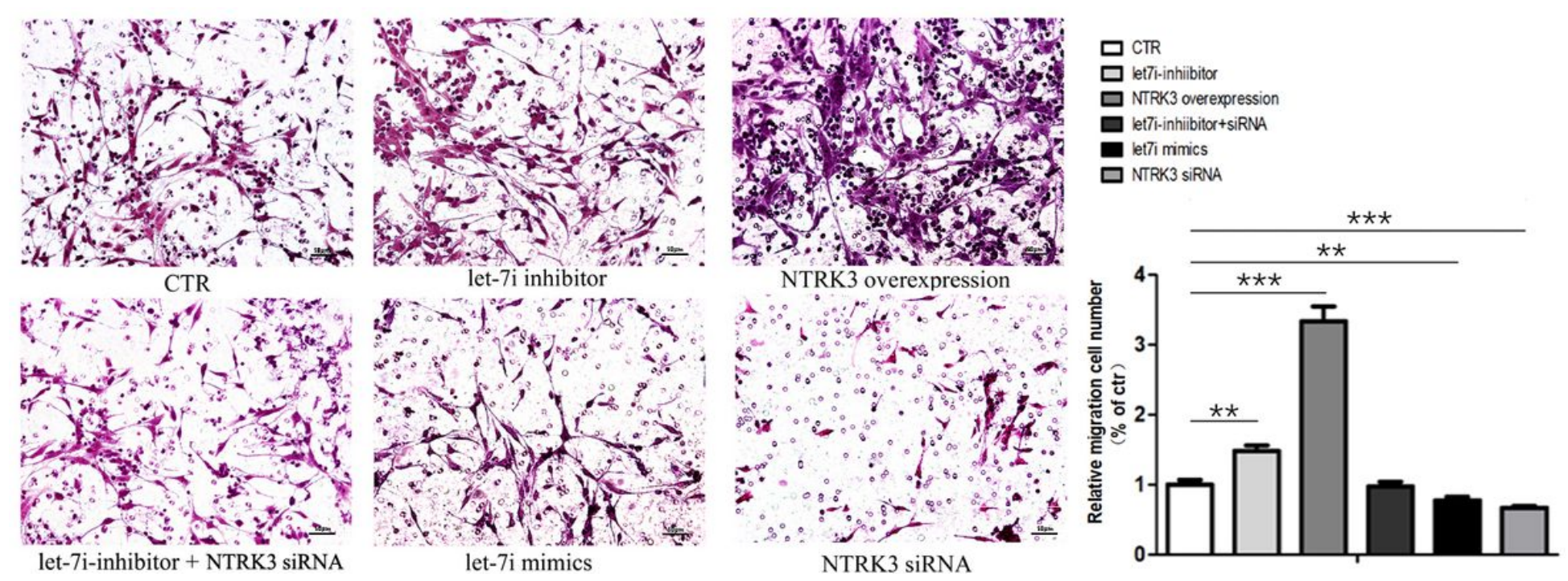

Figure 5

The transwell migration assay indicated an effect of let-7i-5p and NTRK3 on NSC migration in the presence of NT-3. Quantification and representative photomicrographs showing that NTRK3 promotes cell migration. Scale bars: $50 \mu \mathrm{m}$. Data represent mean \pm S.D. from three independent experiments. ** $P<0.01, * \star \star P<0.001$. 
(A)
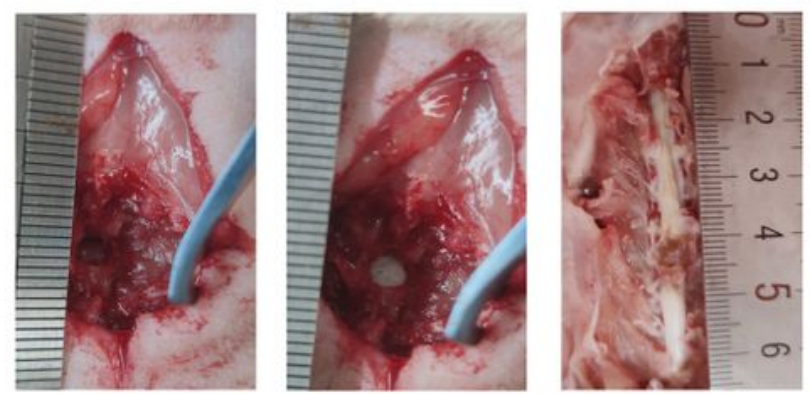

(B)

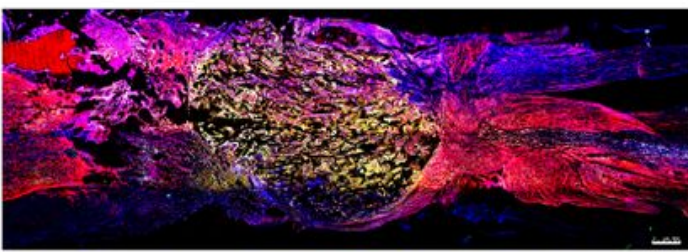

static +NT-3
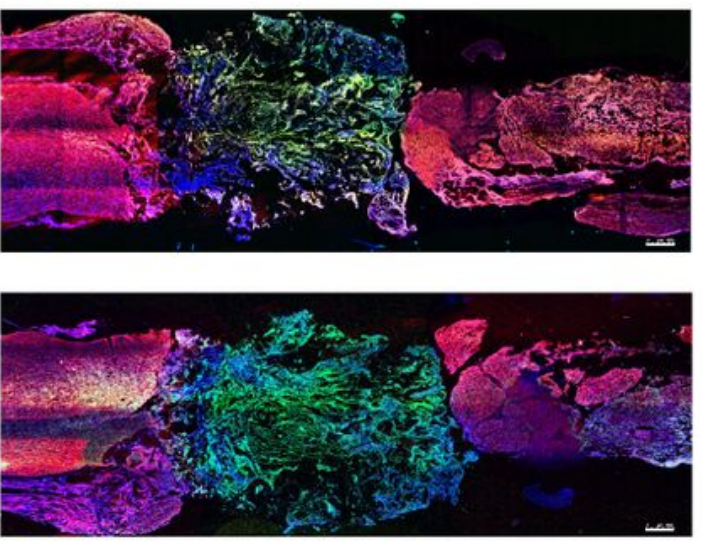

RCCS

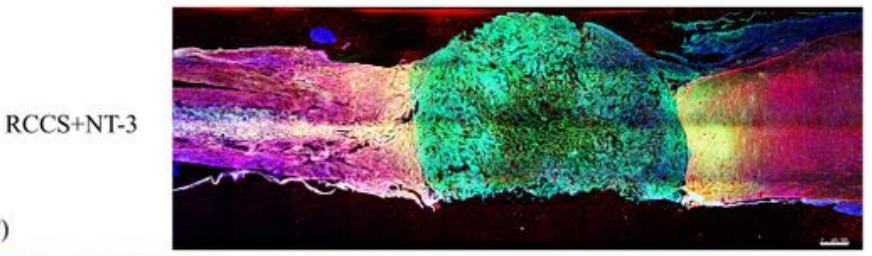

(C)
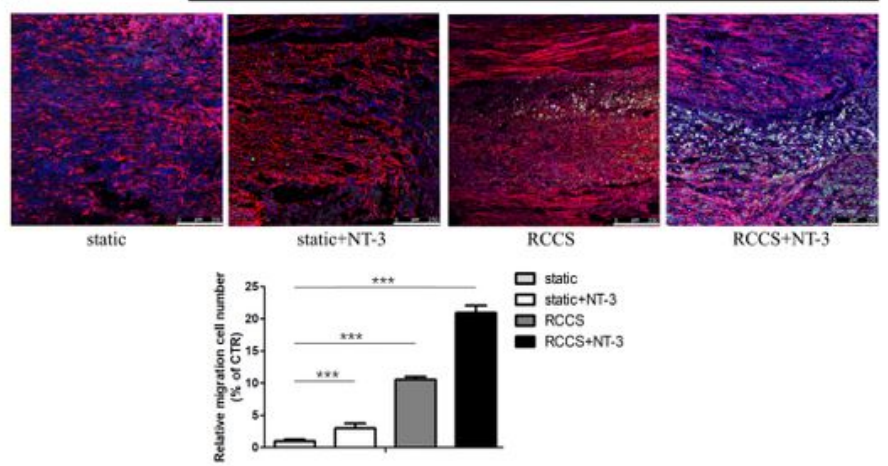

Figure 6

The transplantation of NSCs in rats with spinal cord injury. (A) Spinal cord injuries (with lengths of $3 \mathrm{~mm}$ ) were inflicted in rats (left). Functional collagen sponge scaffolds with or without cells were precisely placed into the injury site (middle). Samples of the spinal cord tissue following perfused $4 \%$ paraformaldehyde fixation (right). (B) Images of immunofluorescence staining of spinal injury sections at 2 weeks from different groups of SCI rats, stained with antibodies against GFAP (red) and with DAPI 
(blue). The green signal represents the GFP-labelled transplanted NSCs. Scale bars: $500 \mu \mathrm{m}$. (C) Enlarged images of the migrated GFP+ cells in the RCCS and RCCS+NT-3 groups. Quantification of the number of migrated cells showing that the NSCs in RCCS+NT-3 group possess the best migratory ability in SCl animals. Scale bars: $250 \mu \mathrm{m}$. Data represent mean \pm S.D. from three independent experiments. ${ }^{* \star *} \mathrm{P}<0.001$. 